\title{
THE GENERIC TRANSFORMATION HAS ROOTS OF ALL ORDERS
}

BY

JONATHAN L. F. KING (GAINESVILLE, FL)

Dedicated to the memory of Anzelm Iwanik

\begin{abstract}
In the sense of the Baire Category Theorem we show that the generic transformation $T$ has roots of all orders (RAO theorem). The argument appears novel in that it proceeds by establishing that the set of such $T$ is not meager - and then appeals to a Zero-One Law (Lemma 2).

On the group $\Omega$ of (invertible measure-preserving) transformations, $\S \mathrm{D}$ shows that the squaring map $\wp: S \mapsto S^{2}$ is topologically complex in that both the locally-dense and locally-lacunary points of $\wp$ are dense (Theorem 23).

The last section, $\S \mathrm{E}$, discusses the relation between $\mathrm{RAO}$ and a recent example of Blair Madore. Answering a question of the author's, Madore constructs a transformation with a square-root chain of each finite length, yet possessing no infinite square-root chain.
\end{abstract}

\section{§A. HISTORY}

A transformation $T$ might have a cartesian square root, $T=S^{\times 2}:=$ $S \times S$, or a composition square root, $T=R^{2}:=R \circ R$; I will henceforth call composition roots just roots. In a paper which had a significant impact on Ergodic theory in the 1970's and 1980's, On the root problem in ergodic theory, [14], Don Ornstein constructed a remarkable transformation $T$ with no roots. Then Dan Rudolph showed, in [15], that the parameters of Ornstein's construction could be tuned to insure that $T$ was prime - no factors- and therefore had no cartesian roots. (This was shown independently by Ken Berg in [2].) Indeed, Rudolph constructed a $T$ with the stronger property of minimal self-joinings, and showed that MSJ implies primeness and trivial commutant. Later work, [11], showed that MSJ followed automatically from $T$ being both mixing and rank-1. The MSJ property, and the more general property of simplicity, have been fruitful for the study of classes of zero-entropy maps, $[16,7]$.

2000 Mathematics Subject Classification: Primary 28D05; Secondary 54E32. 
Ornstein's map has no roots "because" it commutes only with its powers. An entirely different type of rootless $T$ was constructed in [3] via an algebraic automorphism-extension; the commutant of this $T$ is uncountable.

The following overview uses topological terms almost-open, meager, residual (= generic), coarse topology and BaireCat space. These will be defined further below.

Genericity. What happens generically for transformations on a Lebesgue probability space $(\mathbb{I}, \mu)$ ? We ask this question with respect to the standard coarse topology on $\boldsymbol{\Omega}$, where $\boldsymbol{\Omega}$ is the group -under composition- of (invertible measure-preserving) transformations on $\mathbb{I}$.

It follows from the Weak-Closure theorem of [10] that no rank-1 map has a cartesian square root. Since RANK-1 is generic $\left({ }^{1}\right)$, only a meager set of transformations in $\Omega$ can have a cartesian square root.

In contrast, the goal of this article is to show that possessing composition roots is generic. To this end, let $\wp_{e}: \boldsymbol{\Omega} \rightarrow \boldsymbol{\Omega}$ denote the $e$-th-power map $S \mapsto S^{e}$.

(1) RAO THEOREM (Roots of All Orders). The set of transformations with roots of all orders,

$$
\mathrm{RAO}:=\bigcap_{e=2}^{\infty} \wp_{e}(\boldsymbol{\Omega})
$$

is $\boldsymbol{\Omega}$-residual.

It suffices to show that $\wp_{e}(\boldsymbol{\Omega})$ is residual, for each exponent $e$. Most of the ideas appear in the $e=2$ case, so we henceforth let $\wp$ be the squaring map $\wp_{2}$, and endeavor to show that

$$
\text { SQUARES }:=\wp(\boldsymbol{\Omega}) \text { is residual. }
$$

The sequel will reduce this $(\zeta 1)$, in a series of steps, to an assertion $(\zeta 4)$. Here is a roadmap.

\section{OUTLINE}

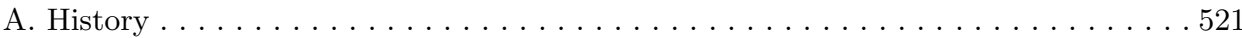

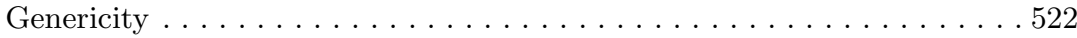

RAO Theorem . . . . . . . . . . . . . . . . . . . . . . . 522

Zero-One Lemma . . . . . . . . . . . . . . . . . . . . . . . . . . . . . . . . . . . 524

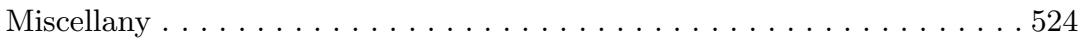

Topological preliminaries . . . . . . . . . . . . . . . . . . . . . . . . . . . . 524

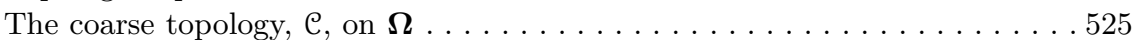

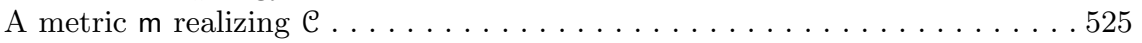

$\left({ }^{1}\right)$ This is implicit in [5, pp. 65-68], and also follows from the Rokhlin lemma. Several equivalent definitions of rank-1 appear in the introduction of [11]. 
The Baire necessities . . . . . . . . . . . . . . . . . . . . . . . 526

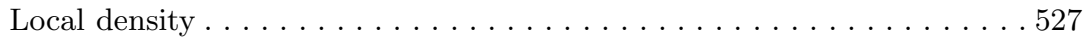

Dougherty's Lemma. . . . . . . . . . . . . . . . . . . . . . . . . . . 527

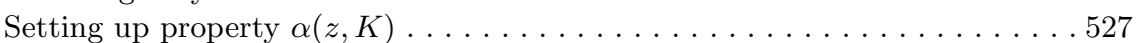

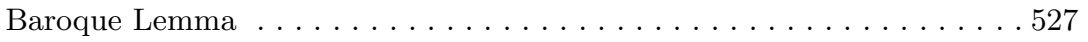

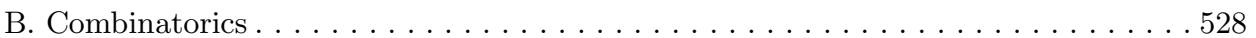

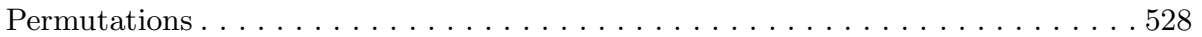

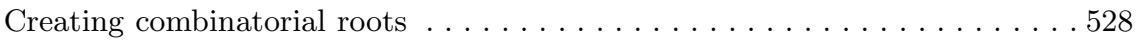

The Weave operation . . . . . . . . . . . . . . . . . . . . . . . 5529

Root Lemma . . . . . . . . . . . . . . . . . . . . . . . . . . . . . . . 529

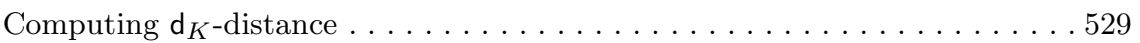

Frequency Lemma . . . . . . . . . . . . . . . . . . . . . . . . . . . . . . . . 529

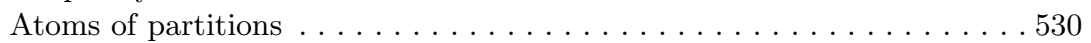

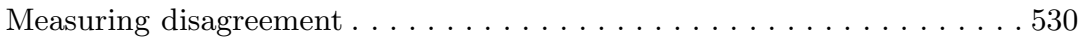

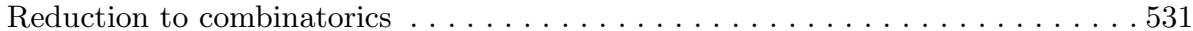

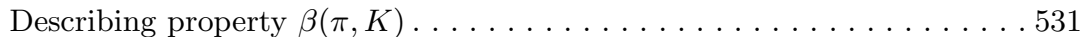

LocDen Combinatorial Lemma . . . . . . . . . . . . . . . . . . . . . 532

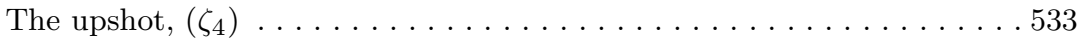

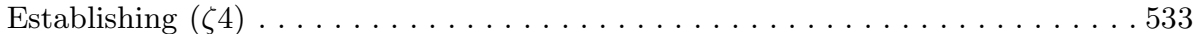

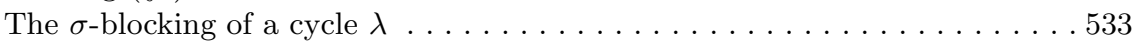

Blocking Lemma . . . . . . . . . . . . . . . . . . . . . . . . 534

Upper-bounding disagreement . . . . . . . . . . . . . . . . . . . . 534

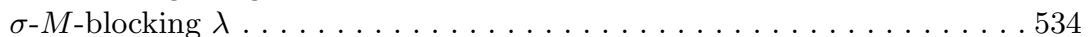

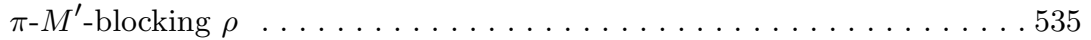

Completing the proof of $(\zeta 4)$ : Picking $\delta \ldots \ldots \ldots \ldots \ldots \ldots \ldots \ldots \ldots \ldots \ldots \ldots \ldots \ldots \ldots$

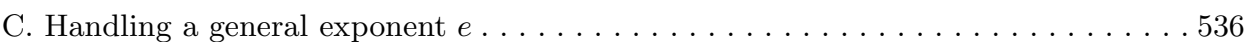

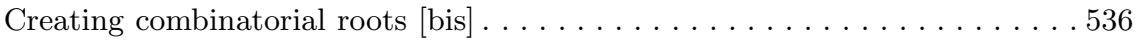

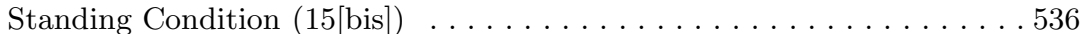

Reflections on the $\operatorname{argument} \ldots \ldots \ldots \ldots \ldots \ldots \ldots$

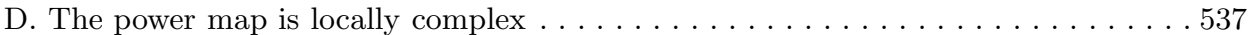

Locally-lacunary . . . . . . . . . . . . . . . . . . . . . . . . . . . . 538

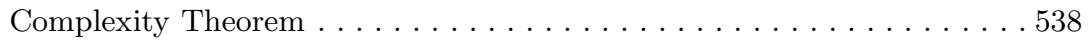

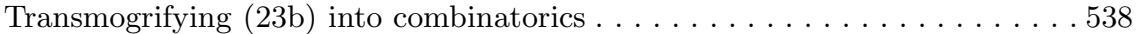

Defining property $\gamma(\pi, K) \ldots \ldots \ldots \ldots$. . . . . . . . . . . . . . . . . . . . . . . . . . . . . . .

LocLAC Combinatorial Lemma . . . . . . . . . . . . . . . . . . . . . . 540

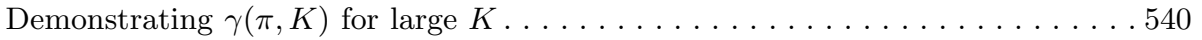

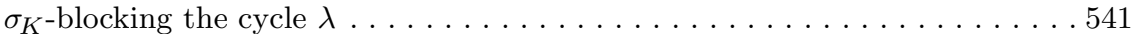

Lower-bounding disagreement . . . . . . . . . . . . . . . . . . . 541

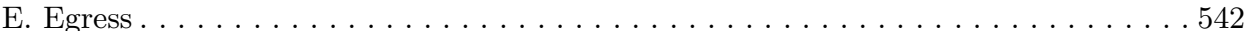

Root chains . . . . . . . . . . . . . . . . . . . . . . . . . . . . .543

Techniques of del Junco and others . . . . . . . . . . . . . . . . 544

Madore's Theorem . . . . . . . . . . . . . . . . . . . . . . . . . . . . . . 545

Conditions implying a $\mathbb{Q}_{\infty}$ extension . . . . . . . . . . . . . . . . 546

Closing thoughts . . . . . . . . . . . . . . . . . . . . . . . 546

A tool that we need is the following special case of the Zero-One Law for genericity. In the terminology of [4], a subset $\mathbf{P} \subset \boldsymbol{\Omega}$ is dynamical if 
(i) It is isomorphism-invariant: Whenever a transformation $T^{\prime}$ is isomorphic to a $T \in \mathbf{P}$, then $T^{\prime}$ itself is in $\mathbf{P}$.

(ii) It is sufficiently measurable: $\mathbf{P}$ is an almost-open subset of $\boldsymbol{\Omega}$.

(2) Zero-One Lemma ([4, p. 232]). Each dynamical property $\mathbf{P} \subset \boldsymbol{\Omega}$ is either meager or residual.

Miscellany. Use " $x:=$ foo" or " $f o o=: x$ " to mean that $f o o$ is the definition of symbol $x$. When defining a term, we use a boldface italic font, whereas just italics indicates emphasis. We use the small-caps font to indicate the set of objects satisfying a property, e.g., RAO, RANK-1, SQUARESand, later, WeAKMixing and LocDen.

Use $A \backslash B$ for the difference of two sets. Use $A \triangle B$ for the symmetric difference $[A \backslash B] \cup[B \backslash A]$. For real numbers $a$ and $b$, let $[a . . b)$ denote the "interval of integers" $[a, b) \cap \mathbb{Z}$.

Employ $K \triangleleft L$ for " $K$ divides $L$ ", and use $L \triangleright K$ for " $L$ is a multiple of $K^{\prime \prime}$.

To indicate a map from a set to itself, we may write $f: X \bigcirc$ instead of $f: X \rightarrow X$.

Topological preliminaries. On a set $X$, a family $\left\{\mathcal{W}_{n}\right\}_{n=1}^{\infty}$ of topologies engenders the coarsest (fewest open sets) topology, $\mathcal{X}$, such that each $\mathcal{W}_{n}$ $\subset \mathcal{X}$. This $X$ is generated by finite intersections $U_{n_{1}} \cap \ldots \cap U_{n_{K}}$, where each $U_{n} \in \mathcal{W}_{n}$.

Suppose further, for each $X$-open set $X^{\prime}$ and point $z \in X^{\prime}$, that the following holds.

(3) For all sufficiently large $n$, there is a $\mathcal{W}_{n}$-open set $U_{n}$ for which $z \in$ $U_{n} \subset X^{\prime}$.

If so, say that "sequence $\left(\mathcal{W}_{n}\right)_{n=1}^{\infty}$ tends to $X$ " and write $\mathcal{W}_{n} \nearrow \mathcal{X}$.

For a pseudo-metric d (a symmetric mapping d: $X \times X \rightarrow[0, \infty)$ satisfying the triangle inequality, but allowing $\mathrm{d}(x, z)=0)$ and point $z \in X$, use $\mathrm{d}-\operatorname{Ball}_{\varepsilon}(z)$ to mean the set of $x$ for which $\mathrm{d}(x, z)<\varepsilon$.

Now suppose that $\mathrm{d}_{n}$ is a pseudo-metric on $X$, with $\mathcal{W}_{n}$ its topology comprising all unions of $\mathrm{d}_{n}$-balls. If each $\mathrm{d}_{n}$ is bounded by 1 , then topology $X$ is realized by pseudo-metric

$$
\mathrm{m}:=\sum_{n=1}^{\infty} \frac{1}{2^{n}} \mathrm{~d}_{n} .
$$

The next two headings will develop the requisite topological notions to prove $(\zeta 1)$. 
The coarse topology, $\mathcal{C}$, on $\boldsymbol{\Omega}$. Since any two non-atomic Lebesgue probability spaces are isomorphic, henceforth take $\mathbb{I}$ to be the half-open interval $[0,1)$ with Lebesgue measure $\mu$. The coarse topology on $\boldsymbol{\Omega}$, call it $\mathcal{C}$, is the topology in which transformations $\tau_{j}$ approach $T$ iff for each measurable set $E \subset \mathbb{I}$ :

$$
\mu\left(\tau_{j}^{-1}(E) \triangle T^{-1}(E)\right) \rightarrow 0, \quad \text { as } j \rightarrow \infty .
$$

Said differently, the coarse topology on $\Omega$ is simply the strong operator topology with each transformation $T \in \boldsymbol{\Omega}$ regarded as a unitary operator on $\mathbb{L}^{2}(\mu)$.

A metric $\mathrm{m}$ realizing $\mathcal{C}$. In the sequel, let interval mean a non-void half-open set $[a, b) \subset \mathbb{I}$. A partition $Q$ decomposes $\mathbb{I}$ into equal-length subintervals,

$$
Q=Q_{k}:=\left\{\left[0, \frac{1}{k}\right),\left[\frac{1}{k}, \frac{2}{k}\right), \ldots,\left[\frac{k-1}{k}, 1\right)\right\},
$$

called the atoms of $Q$. A partition $P$ refines $Q$ if each $Q$-atom $A \in Q$ is some union of $P$-atoms. The formula

$$
\mathrm{d}_{Q}(S, T):=\sum_{A \in Q} \frac{1}{2} \mu\left(S^{-1}(A) \triangle T^{-1}(A)\right)
$$

shows that a partition $Q$ gives rise to a bounded-by-1 pseudo-metric on $\boldsymbol{\Omega}$. Let $\mathcal{W}_{Q}$ denote the $\mathrm{d}_{Q}$-topology on $\boldsymbol{\Omega}$.

A given finite list $E_{1}, \ldots, E_{L} \subset \mathbb{I}$ of (measurable) sets can be $\varepsilon$-approximated, taking any $k$ sufficiently large, by various unions of $Q_{k}$-atoms. Consequently:

$$
\text { Sequence }\left(\mathcal{W}_{Q_{n}}\right)_{n=1}^{\infty} \text { tends to } \mathcal{C} \text {. }
$$

Letting $\mathrm{d}_{n}$ denote $\mathrm{d}_{Q_{n}}$ in (4), the resulting pseudo-metric $\mathrm{m}$ is a metric which realizes the coarse topology on $\boldsymbol{\Omega}$.

(6) Lemma. Our space $(\boldsymbol{\Omega}, \mathrm{C})$ is a Polish (homeomorphic to a complete separable metric space) topological group. In particular, each power map $\wp_{e}$ is continuous.

Proof (sketch). It is not difficult to show that $\Omega$ is complete with respect to the metric

$$
T, S \mapsto \mathrm{m}(T, S)+\mathrm{m}\left(T^{-1}, S^{-1}\right) .
$$

And this metric still realizes $\mathcal{C}$, since the mapping $S \mapsto S^{-1}$ is continuous, as now shown: It suffices to take transformations $\sigma_{j} \rightarrow S$ and fix a set $E$, then establish that $\sigma_{j}(E)$ tends to $S(E)$. But letting $A:=S(E)$, note that

$$
\mu\left(\sigma_{j}(E) \triangle S(E)\right)=\mu\left(E \triangle \sigma_{j}^{-1} S(E)\right)=\mu\left(S^{-1}(A) \triangle \sigma_{j}^{-1}(A)\right),
$$

which certainly goes to zero as $j \rightarrow \infty$. 
To show that the group-multiplication is continuous, we need only measure convergence on a fixed set $A$. To this end, let $\llbracket \tau, \sigma \rrbracket$ abbreviate $\mu\left(\tau^{-1}(A) \triangle \sigma^{-1}(A)\right)$. Fix maps $T$ and $S$, let $E:=T^{-1}(A)$, and consider transformations $\tau_{j} \rightarrow T$ and $\sigma_{j} \rightarrow S$. Evidently

$$
\begin{aligned}
\llbracket \tau_{j} \sigma_{j}, T S \rrbracket & \leq \llbracket \tau_{j} \sigma_{j}, T \sigma_{j} \rrbracket+\llbracket T \sigma_{j}, T S \rrbracket \\
& =\llbracket \tau_{j}, T \rrbracket+\mu\left(\sigma_{j}^{-1}(E) \triangle S^{-1}(E)\right) .
\end{aligned}
$$

And these two terms go to zero, as $j \rightarrow \infty$.

Finally, as detailed in Proposition 10 below, those transformations which permute the atoms of some partition form a set which is $\boldsymbol{\Omega}$-dense and countable. Hence $\boldsymbol{\Omega}$ is separable. (For further discussion, see [5, pp. 62-68].)

The Baire necessities. Recall that a subset $B \subset X$ of a topological space is nowhere-dense if its closure has no interior. Less stringent, $B$ is meager if it equals some countable union of nowhere-dense sets. Finally, a subset $E \subset X$ is residual if its complement $X \backslash E$ is meager.

Say that $X$ is a BaireCat space if the conclusion of the Baire Category Theorem holds in this form:

\section{Each residual subset of $X$ is dense.}

Since $\boldsymbol{\Omega}$ is completely metrizable, it is BaireCat $\left({ }^{2}\right)$.

For the Zero-One Law to apply to Squares, we need to know that SQUARES fulfills the stated measurability condition. Say that a subset $B \subset X$ is almost-open if it is "almost" equal to some open set $U$-in the sense that the symmetric difference $B \triangle U$ is meager. It is straightforward to show that the almost-open sets form a sigma-algebra. A fortiori the open sets are almost-open, so AlmostOpen includes $\left({ }^{3}\right)$ the Borel sigma-algebra. Here is the non-trivial fact that we need.

(7) Theorem. Each analytic set (the forward image of a Borel set, under a continuous map between Polish spaces) is almost-open.

Proof. See [12, pp. 482, 92].

This result comes into play as follows. Since $\wp$ is continuous, the set of pairs $(R, T)$ with $R^{2}=T$ is a closed subset of Polish space $\boldsymbol{\Omega} \times \boldsymbol{\Omega}$. And SQUARES is its continuous image under

$$
\boldsymbol{\Omega} \times \boldsymbol{\Omega} \rightarrow \boldsymbol{\Omega}:(S, T) \mapsto T,
$$

$\left({ }^{2}\right)$ A standard term is Baire, rather than BaireCat; alas, the modifier "Baire" is used inconsistently. It has three distinct meanings in these usages: "a Baire space", "a Baire set", "a set with the property of Baire". Unfortunately a Baire set is not -with the standard terminology - a Baire space in the induced topology.

$\left({ }^{3}\right)$ This inclusion is proper: Computing cardinalities shows that \#ALmostOpen = \#MEAGER $=2^{|\mathbb{R}|}$, whereas \#BOREL only equals $|\mathbb{R}|$. 
the projection map. Thus SquAREs is analytic, hence almost-open. So by Zero-One, we need but establish that

\section{SQUARES is non-meager.}

Our next step is to develop a condition which guarantees non-meagerness of a continuous image.

Local density. Suppose that $f: X \rightarrow \Lambda$ is a map between topological spaces. A point $z \in X$ is locally-dense (with respect to $f$ ) if:

Each neighborhood of $z$ has $f$-image which is dense in some neighborhood of $f(z)$.

Let $\operatorname{LocDen}(f)$ denote the set of locally-dense points. The following neat observation is due to Randall Dougherty.

(8) Dougherty's Lemma. Consider $f: X \rightarrow \Lambda$, a continuous map from a BaireCat space to a topological space. If $\operatorname{LocDeN}(f)$ is $X$-dense, then $f(X)$ is not $\Lambda$-meager.

P r o of. Were $f(X)$ meager, then we could cover it by a union $\bigcup_{n=1}^{\infty} \Gamma_{n}$ of closed sets, each without interior. Thus each $C_{n}:=f^{-1}\left(\Gamma_{n}\right)$ is closed, and $\bigcup_{n=1}^{\infty} C_{n}$ covers $X$.

The interior, $U$, of a $C_{n}$ has its $f$-image inside the closed interiorless set $\Gamma_{n}$, so no point of $U$ is locally-dense. Thus $C_{n}$ has no interior, hence is meager. Consequently $X$ is not BaireCat.

Remark. The same argument shows that the $f$-image of each $X$-residual set is not $\Lambda$-meager.

Setting up property $\alpha(z, K)$. Topologically, the last tool we need is the lemma below. The baroqueness of its formulation is adapted to its use in $\S \mathrm{B}$.

Consider a map between topological spaces,

$$
f:(X, X) \rightarrow(\Lambda, \mathcal{L})
$$

as well as topologies $\mathcal{W}_{n} \nearrow \mathcal{X}$ and topologies $\mathcal{K}_{n} \subset \mathcal{L}$. For a point $z \in X$ and positive integer $K$, define this property:

PROPERTY $\alpha(z, K)$ : Given a $\mathcal{W}_{K}$-open set $U \ni z$ there exists an $\mathcal{L}$-dense set $\Delta \subset \Lambda$ and a $\mathcal{K}_{K}$-open set $\Upsilon \ni f(z)$ so that:

$\left(\alpha^{\prime}\right) \quad f(U) \supset \Upsilon \cap \Delta$.

(9) BARoque Lemma. With notation from immediately above, suppose that $\alpha(z, n)$ holds for infinitely many $n$. Then $z$ is locally-dense for $f$. 
Proof. Fix an arbitrary $X$-open set $Z \ni z$. Among those $n$ fulfilling $\alpha(z, n)$, take $n$ large enough to produce a $\mathcal{W}_{n}$-open set $U$ for which $z \in U \subset$ $Z$. Then take subsets $\Delta, \Upsilon \subset \Lambda$ as $\alpha(z, n)$ provides. A fortiori $\Upsilon$ is $\mathcal{L}$-open, so $\Upsilon \cap \Delta$ is $\mathcal{L}$-dense in $\Upsilon$. Take $\mathcal{L}$-closures; then

$$
\overline{f(Z)} \supset \overline{f(U)} \supset \overline{\Upsilon \cap \Delta} \supset \Upsilon \ni f(z),
$$

as was desired.

\section{$\S B$. COMBINATORICS}

The foregoing discussion will allow us to make "local-density" a purely combinatorial matter. Henceforth, $\mathrm{d}_{k}$ denotes pseudo-metric $\mathrm{d}_{Q_{k}}$ from (5).

Permutations. A permutation of $[0 . . K)$ will be called a $K$-perm. If a $K$-perm $\pi:[0 . . K) \bigcirc$ happens to comprise a single cycle -necessarily of length $K$-then we call $\pi$ a $K$-cycle. Each $K$-perm $\pi$ has an associated interval-exchange map $G_{\pi} \in \boldsymbol{\Omega}$ which rigidly permutes the atoms of partition $Q_{K}$ according to $\pi$. Specifically, letting $A_{\ell}$ be the atom $\left[\frac{\ell}{K}, \frac{\ell+1}{K}\right)$ we set

$$
G_{\pi}(x):=\frac{\pi(\ell)}{K}+\left[x-\frac{\ell}{K}\right], \quad \text { for } x \in A_{\ell} .
$$

Call transformation $G_{\pi}$ a $K$-shuffle, or just a shuffle. Evidently if $T$ is a $K$-shuffle and $K$ divides $L$, then $T$ is also an $L$-shuffle.

As an example, take $K=5$ and $T:=G_{\pi}$, where $\pi$ maps 2 to 0 to 4 to 2 , and $\pi$ exchanges 1 and 3 . Then

$$
\text { (2 } \left.0 \begin{array}{lll}
2 & 4
\end{array}\right)\left(\begin{array}{ll}
1 & 3
\end{array}\right)
$$

is the cycle structure of $\pi$. Call $(*)$ also "the 5 -structure of $T$ ". In contrast, the 10-structure of $T$ splits each cycle into two copies:

$$
\left(\begin{array}{lll}
4 & 0 & 8
\end{array}\right)\left(\begin{array}{lll}
5 & 1 & 9
\end{array}\right)(2 \mathrm{2} 6)(3 \mathrm{3}) \text {. }
$$

If the $K$-structure of $T$ is a single cycle then we call $T$ a $K$-solo. The Rokhlin lemma or [5, p. 65] implies the following.

(10) Proposition. The set of $K$-solos, with $K$ ranging over an infinite set of positive integers, is $\mathcal{C}$-dense in $\boldsymbol{\Omega}$.

Creating combinatorial roots. Given an $L$-perm $\lambda$, say that an $L^{\prime}$-perm $\rho$ is a "combinatorial $n$-th root of $\lambda$ " if $L^{\prime} p L$ and, for the corresponding transformations, $\left[G_{\rho}\right]^{n}$ equals $G_{\lambda}$.

We now construct combinatorial square roots. Consider an $L$-sequence $\mathbf{c}=c_{0} \ldots c_{L-1}$ of numbers. Let $|\mathbf{c}|^{3}$ be the sequence rotated by 3 positions. Thus

$$
|\mathbf{c}|^{3}:=c_{3} c_{4} c_{5} \ldots c_{L-1} c_{0} c_{1} c_{2} .
$$

For $r \in \mathbb{Z}$, define the rotation $\mid \mathbf{c} \iota^{r}$ analogously; so $\mid \mathbf{c} \iota^{0}=\mathbf{c}$. 
A linear expression such as " $2 \mathbf{c}+5$ " shall mean the $L$-sequence $\mathbf{d}$, where each $d_{\ell}:=2 c_{\ell}+5$.

Given two $L$-sequences a and $\mathbf{c}$, let

$$
\mathbf{a}: \mathbf{c}:=a_{0} c_{0} a_{1} c_{1} a_{2} c_{2} \ldots a_{L-1} c_{L-1}
$$

denote their alternation.

The Weave operation. From an $L$-cycle $\lambda$, we can produce a square root of the corresponding $L$-solo $G_{\lambda}$ by cutting its Rokhlin stack into left/right halves, rotating one half (perhaps), and then zig-zagging. We now describe this operation directly on $\lambda$.

Arbitrarily cut cycle $\lambda$ to produce

$$
\begin{aligned}
& \text { an } L \text {-sequence } c_{0} c_{1} c_{2} \ldots c_{L-1} \text {, with each } c_{\ell} \in[0 . . L) \text {; here } \lambda\left(c_{\ell}\right)= \\
& c_{\ell \oplus 1} \text {, where } \oplus \text { denotes addition } \bmod L \text {. }
\end{aligned}
$$

For a "rotation number" $r \in \mathbb{Z}$, define this cycle:

$$
\text { Weave }_{\lambda}(r):=\left(2 \mathbf{c}: 12 \mathbf{c}+1 l^{r}\right) .
$$

The resulting cycle has length $2 L$, and does not depend on where $\lambda$ was cut.

(12) Example. Suppose that $L=7$ and sequence $\mathbf{c}$ is 0246135. Zig-zagging gives this 14-cycle:

$$
\text { Weave }_{\lambda}(0)=(\underline{0} \dot{0} \underline{2} \dot{2} \underline{4} \dot{4} \underline{6} \dot{6} \underline{1} \dot{1} \underline{3} \dot{3} \underline{5} \dot{5}),
$$

where $\underline{c}$ denotes $2 c$, and $\dot{c}$ means $2 c+1$. Rotating the dotted numbers by 4 places produces

$$
\begin{aligned}
& \text { Weave }_{\lambda}(4)=(\underline{0} \dot{1} \underline{2} \dot{3} \underline{4} \dot{5} \underline{6} \dot{0} \underline{1} \dot{2} \underline{3} \dot{4} \underline{5} \dot{6}) \\
& =(034781112125691013) \text {. }
\end{aligned}
$$

For $\rho:=$ Weave $_{\lambda}(r)$, observe that the cycle structure of $\rho^{2}$ is $(2 \mathbf{c})(2 \mathbf{c}+1)$, regardless of what $r$ equals. And since the $\ell$-th atom of partition $Q_{L}$ is the union of atoms $2 \ell$ and $2 \ell+1$ of $Q_{2 L}$, we see that transformation $\left[G_{\rho}\right]^{2}$ equals $G_{\lambda}$.

(13) Root Lemma. For an L-cycle $\lambda$ and rotation number $r \in \mathbb{Z}$, the $2 L$-cycle Weave $_{\lambda}(r)$ is a combinatorial square root of $\lambda$.

Computing $\mathrm{d}_{K}$-distance. We now convert distance between shuffles to a computation directly with permutations. The notation for the following lemma appears anon.

(14) Frequency Lemma. Suppose that $\pi$ is an $K$-perm and $\rho$ is an L-perm, where $L p K$. Then

$$
\mathrm{d}_{K}\left(G_{\pi}, G_{\rho}\right)=\boldsymbol{D}_{\pi}(\rho) .
$$


Atoms of partitions. Enumerate the atoms of $Q_{K}$ as $A_{0}, \ldots, A_{K-1}$ and the atoms of $Q_{L}$ as $C_{0}, \ldots, C_{L-1}$. For the nonce letting $V$ denote the ratio $\frac{L}{K}$, remark that each atom $A_{k}$ is the disjoint union

$$
A_{k}=\bigsqcup_{\ell=V k}^{V k+[V-1]} C_{\ell},
$$

of $V$ consecutive $Q_{L}$-atoms. For an index $\ell$, let $\widehat{\ell}$ be the corresponding value of $k$ in $(*)$. That is,

$$
\widehat{\ell}:=\left\lfloor\ell \cdot \frac{K}{L}\right\rfloor,
$$

where $\lfloor\cdot\rfloor$ is the floor (greatest integer) function.

Measuring disagreement. Use $\boldsymbol{D}_{\pi}(\rho)$ to measure the frequency of discord between $\pi$ and $\rho$, as measured on the partition, $Q_{K}$, that $\pi$ permutes:

$$
\boldsymbol{D}_{\pi}(\rho):=\frac{1}{L} \cdot \#\{\ell \in[0 . . L) \mid \widehat{\rho(\ell)} \neq \pi(\widehat{\ell})\} .
$$

EXAMPle 12 (revisited). Let permutation $\pi$ be the 7 -cycle (0123456). Its square is $\lambda=(0246135)$ of Example 12. The 14-structure of $G_{\pi}$ is

$$
\pi=(024681012)(135791113) .
$$

The cycle $\rho:=$ Weave $_{\lambda}(4)$ of Example 12 is

$$
\rho=(034781112125691013) .
$$

Evidently, $G_{\rho} \neq G_{\pi}$. Although $\mathrm{d}_{14}\left(G_{\rho}, G_{\pi}\right)$ is positive, the $\mathrm{d}_{7}$-distance is zero. To see this, apply the reduction $\ell \mapsto \widehat{\ell}=\left\lfloor\ell \cdot \frac{7}{14}\right\rfloor$ to the preceding two displays. This results in:

$$
\begin{aligned}
& \pi:\left(\begin{array}{l}
0 \\
\rho
\end{array}\right)\left(\begin{array}{l}
0 \\
0
\end{array} 23456\right)(0123456123456),
\end{aligned}
$$

Thus $\boldsymbol{D}_{\pi}(\rho)=0$.

When we apply this observation, in ( $\zeta 4)$, our cycle $\lambda$ will not be $\pi^{2}$, but rather will be a perturbation of $\pi^{2}$.

Proof of (14), the Frequency Lemma. For specificity, take $K=21$. Define transformations

$$
T:=G_{\pi} \quad \text { and } \quad R:=G_{\rho},
$$

and have $P$ denote partition $Q_{21}$.

Given two $P$-atoms, say, $A_{8}$ and $A_{17}$, let $\mathcal{F}$ be the set of $\ell \in[0 \ldots L)$ with $\widehat{\ell}=8$. Enumerate the $Q_{L}$-atoms as $C_{0}, \ldots, C_{L-1}$; then $A_{8}$ is the disjoint 
union $\bigsqcup_{\ell \in \mathcal{F}} C_{\ell}$. Thus

$$
\mu\left(R\left(A_{8}\right) \cap A_{17}\right)=\sum_{\ell \in \mathcal{F}} \mu\left(R\left(C_{\ell}\right) \cap A_{17}\right)=\frac{1}{L} \cdot \#\{\ell \in \mathcal{F} \mid \widehat{\rho(\ell)}=17\} .
$$

Replacing " 17 " by $\pi(8)$ and " $A_{17}$ " by $T\left(A_{8}\right)$ gives this:

$$
\mu\left(R\left(A_{8}\right) \cap T\left(A_{8}\right)\right)=\frac{1}{L} \cdot \#\{\ell \in \mathcal{F} \mid \widehat{\rho(\ell)}=\pi(\widehat{\ell})\} .
$$

Substituting $k$ for " 8 ", then summing over all $k$ in $[0 . .21)$ yields that

$$
\sum_{A \in P} \mu(R(A) \cap T(A))=\frac{1}{L} \cdot \#\{\ell \in[0 . . L) \mid \widehat{\rho(\ell)}=\pi(\widehat{\ell})\} .
$$

With $B$ denoting $T(A)$, subtract each side of $*$ from 1 to arrive at this:

$$
\begin{aligned}
\boldsymbol{D}_{\pi}(\rho) & =1-\sum_{B \in P} \mu(R(A) \cap B)=\sum_{B}[\mu(B)-\mu(R(A) \cap B)] \\
& =\sum_{B} \frac{1}{2} \mu(R(A) \triangle B) ;
\end{aligned}
$$

this last, since $R(A)$ and $B$ have the same $\mu$-mass. The denouement, by applying $R^{-1}$, is that

$$
\boldsymbol{D}_{\pi}(\rho)=\sum_{B \in P} \frac{1}{2} \mu\left(T^{-1}(B) \triangle R^{-1}(B)\right),
$$

which is indeed the definition of $\mathrm{d}_{K}(T, R)$.

Reduction to combinatorics. Here is the standing condition which is in force for the remainder of $\S \mathrm{B}$.

We have integers $K \ngtr J$, with $J$ odd, and have a $J$-solo $T$. Necessarily, $T$ equals some shuffle $G_{\pi}$, where $\pi:[0 . . K) \bigcirc$ is a permutation comprising $\frac{K}{J}$ many $J$-cycles. Define

$$
\sigma:=\pi^{2}=\pi \circ \pi \quad \text { and } \quad S:=T^{2} \stackrel{\text { note }}{=} G_{\sigma},
$$

the corresponding squares.

Since $J$ is odd, note that permutation $\sigma$, like $\pi$, is made up of $\frac{K}{J}$ many cycles, each of length $J$.

Courtesy of (10), the collection of odd-solos is $\boldsymbol{\Omega}$-dense. Consequently: (ל3) If each odd-solo is locally-dense for $\wp$, then SquARES is $\boldsymbol{\Omega}$-residual. This follows from $(8)$ and $(\zeta 2)$.

Describing property $\beta(\pi, K)$. Let $(\times K)$-cycles be the infinite family of cyclic permutations $\lambda:[0 . . L) \ominus$ taken over all lengths $L=K, 2 K, 3 K, \ldots$ 
Here is a combinatorial property that the $\pi, K$ pair might have.

Property $\beta(\pi, K)$ : Given $\varepsilon$ there is a $\delta>0$ so that for each $(\times K)$-cycle $\lambda$ :

$\left(\beta^{\prime}\right) \quad$ There is a combinatorial square-root $\rho$, of $\lambda$, for which

$$
\boldsymbol{D}_{\sigma}(\lambda)<\delta \Longrightarrow \boldsymbol{D}_{\pi}(\rho)<\varepsilon .
$$

Roughly: "Each perturbation $\lambda$ of the square of $\pi$ has a combinatorial square root close to $\pi . "$

(16) LocDen Combinatorial Lemma. If each pair $\pi$ and $K$ has property $\beta(\pi, K)$, then Squares is residual in $\boldsymbol{\Omega}$.

Proof. Letting $T$ denote $G_{\pi}$, we will first show that

$$
\beta(\pi, K) \Longrightarrow \alpha(T, K),
$$

by letting $\mathrm{d}$ mean $\mathrm{d}_{K}$, and then applying Property $\alpha$ to the squaring map via the correspondence below.

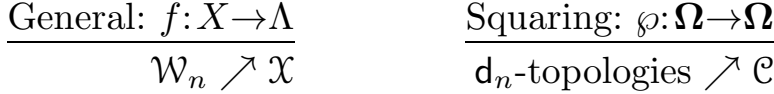

$$
\begin{aligned}
& \mathcal{K}_{n} \subset \mathcal{L} \quad \mathrm{d}_{n} \text {-topology } \subset \mathcal{C} \\
& U, \Upsilon \quad \mathrm{d}-\operatorname{Ball}_{\varepsilon}(T), \mathrm{d}-\operatorname{Ball}_{\delta}(S)
\end{aligned}
$$

Establishing $\left(16^{\prime}\right)$. A d-open set $U \ni T$ may freely be shrunk to some d-Ball ${ }_{\varepsilon}(T)$, for a sufficiently Lilliputian $\varepsilon$. Property $\beta(\pi, K)$ produces a number $\delta$ for which $\left(\beta^{\prime}\right)$ holds. Happily,

$$
\Delta:=\left\{G_{\lambda} \mid \lambda \text { is a }(\times K) \text {-cycle }\right\}
$$

is $\mathcal{C}$-dense, thanks to (10). By definition,

$$
\Upsilon:=\mathrm{d}-\operatorname{Ball}_{\delta}(S)
$$

is a d-open neighborhood of $\wp(T)$.

If we use the Frequency Lemma twice, $\left(\beta^{\prime}\right)$ says that for each $(\times K)$-cycle $\lambda$,

$$
G_{\lambda} \in \Upsilon \Longrightarrow G_{\rho} \in \mathrm{d}-\operatorname{Ball}_{\varepsilon}(T) .
$$

Thus $\Delta \cap \Upsilon \subset \wp(U)$, as required by $\left(\alpha^{\prime}\right)$.

Second step. Given that each $\beta(\pi, K)$ holds, we now know that each $\alpha(T, K)$ holds. If we fix $T$ and vary $K$ over the multiples of $J$, the Baroque Lemma guarantees that $T$ is locally-dense for $\wp$. Courtesy of $(\zeta 3)$ then, SqUARES is $\boldsymbol{\Omega}$-residual. 
The upshot, ( $\zeta 4)$. We have navigated from $\zeta 1$ to this becoming our goal:

$$
\begin{aligned}
& \text { Given } \varepsilon \text { there is a } \delta \text { so that for each } L p K \text { and each } L \text {-cycle } \lambda \text { : } \\
& \qquad \boldsymbol{D}_{\sigma}(\lambda)<\delta \Longrightarrow \boldsymbol{D}_{\pi}(\rho)<2 \varepsilon \\
& \text { where } \rho \text { is the } 2 L \text {-cycle Weave }{ }_{\lambda}\left(\frac{J+1}{2}\right) \text {. }
\end{aligned}
$$

The above assertion is a specification of property $\beta(\pi, K)$, since for an arbitrary integer $r$, the cycle $\rho:=$ Weave $_{\lambda}(r)$ is a combinatorial square root of $\lambda$.

Establishing ( $\zeta 4)$. Given a $K$-perm $\sigma$ and an $L$-cycle $\lambda$, with $L \triangleright K$, we ask: How does frequency-of-disagreement behave relative to the Weave operation?

The results that we need, (18) and (12), arise from substrings of $\lambda$ which look like pieces of $\sigma$.

The $\sigma$-blocking of a cycle $\lambda$. Cut $\lambda$ to produce a sequence $\mathbf{b}$, with each number $b_{\ell} \in[0 . . L)$ and with $\lambda\left(b_{\ell}\right)=b_{\ell \oplus 1}$; here, $\oplus$ means addition mod $L$. Note that from its definition, $\left(14^{\prime}\right)$, computing $\boldsymbol{D}_{\sigma}(\lambda)$ does not require all the information in $\mathbf{b}$. Indeed

$$
\boldsymbol{D}_{\sigma}(\lambda) \stackrel{\text { note }}{=} \frac{1}{L} \cdot \#\left\{\ell \in[0 . . L) \mid c_{\ell \oplus 1} \neq \sigma\left(c_{\ell}\right)\right\}
$$

where $\mathbf{c}$ is the $Q_{K}$-name of $\mathbf{b}$; that is,

$$
c_{\ell}:=\widehat{b_{\ell}} \stackrel{\text { note }}{=}\left\lfloor c_{\ell} \cdot \frac{K}{L}\right\rfloor .
$$

At this juncture, let $\boldsymbol{A} \subset[0 . . L)$ denote the positions of agreementthe set of indices $\ell$ such that $c_{\ell \oplus 1}=\sigma\left(c_{\ell}\right)$; thus $\# \boldsymbol{A} / L$ equals $1-\boldsymbol{D}_{\sigma}(\lambda)$. Decompose $\boldsymbol{A}$ into a disjoint union,

$$
\boldsymbol{A}=\bigsqcup_{n=1}^{N}\left[\ell_{n} . . r_{n}\right)
$$

of half-open intervals (of integers), satisfying $0 \leq \ell_{1}<r_{1}<\ell_{2}<r_{2}<\ldots<$ $r_{N} \leq L$. Each interval $[\ell . . r)$ is called a $\sigma$-block of $\lambda$. This decomposition is unique $\left({ }^{4}\right)$.

$\left({ }^{4}\right)$ There is an exceptional case: When $\boldsymbol{D}_{\sigma}(\lambda)$ is zero, $\boldsymbol{A}$ is the entire cycle $[0 \ldots L)$ and so $\boldsymbol{A}$ does not break into intervals. Since this exceptional case makes the subsequent estimate even better, we can safely ignore it.

With $\boldsymbol{D}_{\sigma}(\lambda)$ positive, we can have chosen to cut $\lambda$ so that no $\sigma$-block "wraps around" the end of $\mathbf{c}$; that is, so that $c_{0} \neq \sigma\left(c_{L-1}\right)$. Once $\mathbf{c}$ has been so chosen, the decomposition is unique. 
Given a positive integer $M$, call a $\sigma$-block $[\ell . . r)$ a $\sigma$-M-block if its length dominates $M$; that is, if $r-\ell \geq M$. Thus the " $\sigma$-M-blocking of $\lambda$ " comprises all the $\sigma$-M-blocks. Let

$$
\mu(\sigma-M \text {-blocks on } \lambda)
$$

denote the probability that an index in $[0 . . L)$ is in some $\sigma$ - $M$-block. Evidently $M=0$ yields the full $\sigma$-blocking, and so

$$
\mu(\sigma \text {-0-blocks on } \lambda)=1-\boldsymbol{D}_{\sigma}(\lambda),
$$

by definition.

(18) Blocking Lemma. With $\sigma, \lambda, L$ and $M$ as above,

(a) $1-\boldsymbol{D}_{\sigma}(\lambda) \geq \mu(\sigma$-M-blocks on $\lambda)$.

(b) $\mu(\sigma-M$-blocks on $\lambda) \geq 1-M \boldsymbol{D}_{\sigma}(\lambda)$.

Pr o of of (b). A miracle occurs: Suppose that every position $r \in[0 . . L)$ of disagreement, $c_{r \oplus 1} \neq \sigma\left(c_{r}\right)$, happens to be the end of a $\sigma$-block $[\ell . . r)$ whose length, $r-\ell$, is exactly $M-1$. In this one case, the quantity $1-$ $\mu(\sigma$ - $M$-blocks $)$ is just large enough to equal $M$ times the probability of a disagreement. So

$$
1-\mu(\sigma-M \text {-blocks on } \lambda) \leq M \cdot \boldsymbol{D}_{\sigma}(\lambda)
$$

is the general non-miraculous assertion.

Upper-bounding disagreement. To establish $(\zeta 4)$, there is no loss of generality in taking particular values for the parameters of our standing condition (15) and so we fix

$$
J=7 \quad \text { and } \quad K=21 .
$$

In consequence, $\pi$ comprises three 7 -cycles.

For specificity, take one of the 7-cycles to be

$$
0 \rightarrow 1 \rightarrow 2 \rightarrow 3 \rightarrow 4 \rightarrow 5 \rightarrow 6
$$

(and $6 \rightarrow 0$ ). The corresponding 7 -cycle of $\sigma$ is

$$
0 \rightarrow 2 \rightarrow 4 \rightarrow 6 \rightarrow 1 \rightarrow 3 \rightarrow 5 .
$$

Given an $L$-cycle $\lambda$ with $\boldsymbol{D}_{\sigma}(\lambda)$ small, define its $Q_{K}$-name $\mathbf{c}$ as we did in the (17) paragraph.

$\sigma$-M-blocking $\lambda$. Having frozen a value for $M$, there are three "types" of $\sigma$ - $M$-block on $\lambda$; one for each of the three 7-cycles of $\sigma$. Suppose that $[\ell . . \ell+M)$ is a $\sigma-M$-block of type (19). That means, letting $\mathbf{s}$ denote the sequence 0246135 , that

$$
\mathbf{c}_{(\ell . \ell \ell+M)}=\underbrace{\mathbf{s s s s s s s} \cdots \mathbf{s s s}}_{M \text { positions }},
$$


where this $\mathbf{s} \cdots \mathbf{s}$ represents a concatenation of copies of $\mathbf{s}$, possibly ending (starting) with an initial (terminal) segment of s. Such segments have no effect on the observation below and so, for simplicity, the notation below presumes that "s $\cdots \mathbf{s}$ " is exactly a concatenation of copies of $\mathbf{s}$.

Thanks to this presumption, the last position of $\mathbf{s} \cdot \mathbf{s}$ is occupied by "5". This is a "position of agreement", so " 5 " must be followed by "0". We will write this trailing zero in slanted and enlarged font. Thus, along the name c, we witness occurrences of

$$
\underbrace{? \text { sssssss } \cdots \operatorname{sss} 0}_{M+2 \text { positions }}
$$

where "?" indicates an unknown symbol in [0..21).

$\pi$ - $M^{\prime}$-blocking $\rho$. Since $\frac{J+1}{2}=\frac{7+1}{2}=4$, we consider $\rho:=$ Weave $_{\lambda}(4)$. How does the $M$-block $\mathbf{s} \cdots \mathbf{s}$ appear on the $\rho$ cycle?

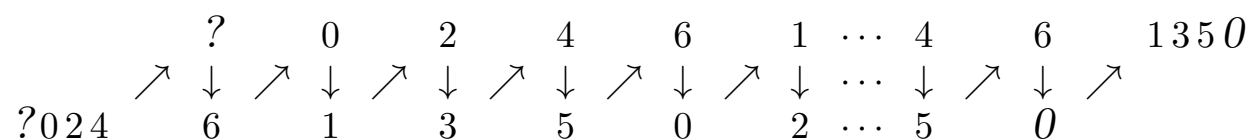

Figure 20. Part of the $Q_{21}$-name of $\rho$ : The upper line shows the $M+2$ string ?s $\cdots \mathbf{s} 0$ which, in the lower line, is shifted left by 4 positions. The arrows show how $\rho$ weaves between the two lines.

Figure 20 shows part of the $\operatorname{Weave}_{\lambda}(4)$ cycle. The $\sigma$ - $M$-block upstairs zips together with the $\sigma$ - $M$-block downstairs to form a $\pi$-block of length at least $2 M-J$.

The upshot is that for $M$ an arbitrary positive integer,

$$
\mu\left(\pi \text { - } M^{\prime} \text {-blocks on } \rho\right) \geq \frac{M^{\prime}}{2 M} \cdot \mu(\sigma \text { - } M \text {-blocks on } \lambda),
$$

where $M^{\prime}$ is $2 M-J$.

Completing the proof of ( $\zeta 4)$ : Picking $\delta$. Since $J, K$ and $\varepsilon$ are known in advance, we can take $M$ sufficiently Brobdingnagian that

$$
\frac{J}{2 M}<\varepsilon ; \text { then let } \delta:=\frac{\varepsilon}{M} .
$$

Consider now an $L$-cycle $\lambda$, with $L p K$ and with $\boldsymbol{D}_{\sigma}(\lambda)<\delta$. Courtesy of $(22,21)$ above,

$$
\mu(\pi \text {-blocks on } \rho) \geq[1-\varepsilon] \cdot \mu(\sigma \text {-M-blocks on } \lambda) .
$$

Furthermore,

$$
\mu(\sigma-M \text {-blocks on } \lambda) \geq 1-M \boldsymbol{D}_{\sigma}(\lambda) \geq 1-\varepsilon,
$$


by (18b) and (22). Together, the two preceding displayed inequalities yield that

$$
1-\boldsymbol{D}_{\pi}(\rho) \geq[1-\varepsilon][1-\varepsilon] \geq 1-2 \varepsilon .
$$

Consequently $2 \varepsilon \geq \boldsymbol{D}_{\pi}(\rho)$, which establishes $(\zeta 4)$ and completes the proof that SQUARES is residual in $\boldsymbol{\Omega}$.

\section{$\S$ C. HANDLING A GENERAL EXPONENT $e$}

We sketch, for a general exponent $2 \leq e<\infty$, the argument that $\wp_{e}(\boldsymbol{\Omega})$ is residual. For simplicity of notation, however, we will take $e=17$; so each "16", below, represents " $e-1$ ".

Say that a positive integer $J$ is $\operatorname{good}$ if $J \equiv-1$, modulo 17 . Here, the good cycles will play the role that the odd cycles played in $\S \mathrm{B}$.

Creating combinatorial roots [bis]. Handed 17 sequences, $\mathbf{a}, \mathbf{b}, \ldots, \mathbf{c}$, each of length $L$, define their alternation $\mathbf{a}: \mathbf{b}: \ldots: \mathbf{c}$ to be the sequence

$$
a_{0} b_{0} \ldots c_{0} a_{1} b_{1} \ldots c_{1} a_{2} b_{2} \ldots c_{2} \cdots a_{L-1} b_{L-1} \ldots c_{L-1}
$$

whose length is $17 \mathrm{~L}$.

Given an $L$-cycle $\lambda$, cut it to produce an $L$-sequence c, as in (11). As before, a linear expression such as " $17 \mathbf{c}+5$ " means the $L$-sequence whose $\ell$-th number is $17 c_{\ell}+5$. For arbitrary rotation numbers $r_{1}, \ldots, r_{16}$, define

$$
\rho:=\text { Weave }_{\lambda}\left(r_{1}, r_{2}, \ldots, r_{16}\right)
$$

to be the cycle

$$
\left(17 \mathbf{c}: 117 \mathbf{c}+1 l^{r_{1}}: 117 \mathbf{c}+\left.2\right|^{r_{2}}: \ldots: 117 \mathbf{c}+\left.16\right|^{r_{16}}\right),
$$

whose length is $17 \mathrm{~L}$. Independent of what the rotation numbers are, the cycle structure of the composition $\rho^{17}$ is

$$
(17 \mathbf{c})(17 \mathbf{c}+1)(17 \mathbf{c}+2) \ldots(17 \mathbf{c}+16) .
$$

Thus transformation $\left[G_{\rho}\right]^{17}$ indeed equals $G_{\lambda}$, so permutation $\rho$ is a combinatorial 17-th root of $\lambda$.

Standing Condition (15[bis]). We set $\sigma:=\pi^{17}$ and $S:=T^{17}$. As before, $\pi$ comprises $\frac{K}{J}$ many $J$-cycles; and so does $\sigma$, since $J$ is relatively prime to 17 . We need but establish this version of $(\zeta 4)$ : 
$(\zeta 4[\mathrm{bis}])$

Given $\varepsilon$ there is a $\delta$ so that for each $L p K$ and each $L$-cycle $\lambda$ :

$\boldsymbol{D}_{\sigma}(\lambda)<\delta \Longrightarrow \boldsymbol{D}_{\pi}(\rho)<2 \varepsilon$

where $\rho$ is the $17 L$-cycle

Weave $_{\lambda}(r, r, r, \ldots, r)$

with $r:=\frac{J+1}{17}$.

With this value of $r$, the reader may convince himself that the following analog of inequality (21) succeeds: For $M$ arbitrary,

$$
\mu\left(\pi-M^{\prime} \text {-blocks on } \rho\right) \geq \frac{M^{\prime}}{17 M} \cdot \mu(\sigma-M \text {-blocks on } \lambda),
$$

with $M^{\prime}:=17 M-F$, where $F$ is some number which depends neither on $L$ nor $M$. A straightforward estimate $\left({ }^{5}\right)$ allows $F=[J+1] \cdot 17$.

The final step is to grab an $M$ sufficiently large that

$$
\frac{F}{17 M}<\varepsilon ; \text { then let } \delta:=\frac{\varepsilon}{M} \text {. }
$$

As before $1-\boldsymbol{D}_{\pi}(\rho) \geq 1-2 \varepsilon$, which delivers the goods on ( $\zeta 4$ [bis] $)$.

Reflections on the argument. The combinatorics used to show $\wp_{2}(\boldsymbol{\Omega})$ residual are elementary. The one non-elementary tool was $(7)$, that analytic sets are almost-open. We could do without this theorem if we had a "yes" to this question.

(Q1) Question. Is $\wp_{2}(\boldsymbol{\Omega})$, the set of Squares, a Borel subset of $\boldsymbol{\Omega}$ ?

In the more general setting of a Polish semigroup, there is an example where the answer is known to be "no". Humke and Laczkovich showed, in the Polish semigroup (under composition) of continuous functions from $[0,1] \odot$, that the set of composition squares $g \circ g$ is not Borel. (See [6]. Also see Beleznay [1].)

\section{$\S D$. THE POWER MAP IS LOCALLY COMPLEX}

Having shown that $\operatorname{LocDen}\left(\wp_{e}\right)$ is dense in $\boldsymbol{\Omega}$, one would be singularly incurious to not inquire about density of its complement.

It turns out that the tools already developed are sufficient to show that $\wp_{e}$ is "locally complex" in the sense of (23ab), below. Part (23a) is simply an embellishment of what we already know.

$\left({ }^{5}\right)$ In general, $F=[J+1] e$ works. A fastidious analysis would justify $F=[J-2][e-1]$, once $M>J+1$. 
Use $\sqrt[e]{T}$ to denote the collection of $e$-th roots of $T$, i.e., the closed set $\wp_{e}^{-1}(T)$.

Locally-lacunary. With respect to a mapping $f: X \rightarrow \Lambda$, a point $z \in X$ is locally-lacunary if it is not locally-dense. Equivalently: There exists a

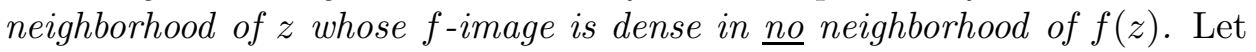
$\operatorname{LOCLAC}(f)$ denote the set of locally-lacunary points of $f$.

(23) Complexity Theorem. For each integer $e \in[2 . . \infty)$ and transformation $T$, the set $\sqrt[e]{T}$ is closed and nowhere-dense in $\boldsymbol{\Omega}$. Furthermore

(a) $\operatorname{LocDen}\left(\wp_{e}\right)$ is residual. Indeed, it is a dense $\mathcal{G}_{\delta}$-subcollection of $\boldsymbol{\Omega}$.

(b) LocLAC $\left(\wp_{e}\right)$, while meager, is dense.

REMARK. Since $\sqrt[e]{T}$ is closed, that it is nowhere-dense follows immediately from noting that WEAKMixing and Rotations are disjoint families, each of which is $\boldsymbol{\Omega}$-dense and is sealed under taking $e$-th roots. (With $\oplus$ meaning addition mod 1 , the set RotATIONS comprises all transformations isomorphic to some rotation $x \mapsto x \oplus r$, for some rotation number $r \in \mathbb{R}$.)

Since $\operatorname{LocDen}\left(\wp_{e}\right)$ is $\boldsymbol{\Omega}$-dense, (23a) follows from this general assertion: (24) Lemma. Suppose $f: X \rightarrow \Lambda$ is continuous map from a metrizable space $X$ (not necessarily separable) to a topological space $\Lambda$. Then $\operatorname{LocDEN}(f)$ is a $\mathcal{G}_{\delta}$-subset of $X$.

Proof. For a number $\alpha>0$, let $U_{\alpha}$ comprise those points $z \in X$ such that: There exists a positive $\varepsilon<\alpha$ for which $f\left(\operatorname{Ball}_{\varepsilon}(z)\right)$ is dense in some neighborhood of $f(z)$.

Since $\operatorname{LOCDEN}(f)=\bigcap_{\alpha \searrow 0} U_{\alpha}$, it will do to simply show that $U_{\alpha}$ is open. For demonstrating openness at a point $z \in U_{\alpha}$, take an $\varepsilon<\alpha$, then an open set $\Upsilon \ni f(z)$ such that $f\left(\operatorname{Ball}_{\varepsilon}(z)\right)$ is dense in $\Upsilon$. Necessarily, the intersection

$$
I:=\operatorname{Ball}_{\alpha-\varepsilon}(z) \cap f^{-1}(\Upsilon)
$$

is an open neighborhood of $z$. We need but show that $I \subset U_{\alpha}$, as follows.

Fix a point $y \in I$ and let $r:=\operatorname{dist}(y, z)$. Then

$$
\operatorname{Ball}_{r+\varepsilon}(y) \supset \operatorname{Ball}_{\varepsilon}(z),
$$

and so the $f$-image of $\operatorname{Ball}_{r+\varepsilon}(y)$ is dense in $\Upsilon$. Therefore $y \in U_{\alpha}$, since $r+\varepsilon<\alpha$.

Transmogrifying (23b) into combinatorics. Following the strategy of $\S \mathrm{B}$, let us find a statement about permutations that will imply (23b). Also as earlier, since all the ideas appear in the $e=2$ case we let $\wp$ mean $\wp_{2}$ from now on. 
It was essential in $\S \mathrm{B}$ that $J$ was odd. Here, in $\S \mathrm{B}$ it is essential that $J$ be even. Fix any large even number $J$ and a $J$-cycle $\pi$. Let $\sigma:=\pi^{2}$ and have $U$ denote this ball:

$$
U:=\operatorname{Ball}_{\frac{1}{3}}\left(G_{\pi}\right),
$$

where the pseudo-metric used is $\mathrm{d}_{J}$. We will show that

$\left(25^{\prime}\right) \quad$ The $\wp$-image of $U$ fails to be dense in each and every neighborhood, $\Upsilon$, of $G_{\sigma}$.

Using $H:=J / 2$ to denote half of $J$, write our $J$-cycle $\pi$ as

$$
(\underbrace{\mathrm{a}_{1} \mathrm{~b}_{1} \mathrm{a}_{2} \mathrm{~b}_{2} \mathrm{a}_{3} \mathrm{~b}_{3} \cdots \mathrm{a}_{H} \mathrm{~b}_{H}}_{\text {reordering of the numbers }[0 \ldots J)}) .
$$

Its square, $\sigma$, has cycle structure $(\boldsymbol{\alpha})(\boldsymbol{\beta})$, two $H$-cycles, where

$$
\boldsymbol{\alpha}:=\mathrm{a}_{1} \mathrm{a}_{2} \mathrm{a}_{3} \ldots \mathrm{a}_{H} \text { and } \boldsymbol{\beta}:=\mathrm{b}_{1} \mathrm{~b}_{2} \mathrm{~b}_{3} \ldots \mathrm{b}_{H}
$$

We now perturb permutation $\sigma$ to a nearby cycle, $\sigma_{K}$.

For each integer $K \triangleright J$, define a $K$-cycle $\sigma_{K}$ to be (AB), where

$$
\mathbf{A}:=\underbrace{\boldsymbol{\alpha} \boldsymbol{\alpha} \boldsymbol{\alpha} \cdots \boldsymbol{\alpha}}_{\frac{K}{J} \text { copies }} \text { and } \mathbf{B}:=\underbrace{\boldsymbol{\beta} \boldsymbol{\beta} \boldsymbol{\beta} \cdots \boldsymbol{\beta}}_{\frac{K}{J} \text { copies }} .
$$

(26) Lemma. In the coarse topology, $G_{\sigma_{K}} \rightarrow G_{\sigma}$ as $K \rightarrow \infty$.

Pro of (sketch). Since the $\mathrm{d}_{N}$-topologies $\nearrow \mathcal{C}$, we need but establish that

$$
\lim _{K \rightarrow \infty} \mathrm{d}_{N}\left(G_{\sigma_{K}}, G_{\sigma}\right)=0
$$

for each $N$ in some sequence $N \rightarrow \infty$; so fix an $N \ngtr J$. Since $\mathrm{d}_{K} \nearrow \mathcal{C}$, we only need prove $(*)$ for, say, those values $K \triangleright N$. Write $K=k N$; then

$$
\mathrm{d}_{N}\left(G_{\sigma_{K}}, G_{\sigma}\right) \leq \frac{1}{k \cdot \operatorname{Length}(\alpha)}=\frac{1}{k H},
$$

courtesy the Frequency Lemma argument.

Defining property $\gamma(\pi, K)$. In what follows, parameters $J, \pi$ and $K$ are implicit. We write $\sigma$ for $\pi^{2}$. Phrases such as "For each/every/all $\rho \ldots$.. shall mean: "For each $L \ngtr K$ and each $L$-cycle $\rho$..." Lastly, $\lambda$ is another name for $\rho^{2}$.

Property $\gamma(\pi, K)$ : There exists a positive $\delta$ so that, for each $\rho$ :

$$
\text { If } \boldsymbol{D}_{\sigma_{K}}(\lambda)<\delta \text {, then } \boldsymbol{D}_{\pi}(\rho) \geq \frac{1}{3} \text {. }
$$


Remark. The value $\frac{1}{3}$, here and in (25), is conceptually $\frac{1}{2}$. We use $\frac{1}{3}$ to allow room for edge effects in the estimate to follow.

(27) Loclac Combinatorial Lemma. If $\gamma(\pi, K)$ holds for all large (or just infinitely many) $K \triangleright J$, then transformation $G_{\pi}$ is locally-lacunary for the squaring map.

P r o of. We verify $\left(25^{\prime}\right)$. Let $\delta(\cdot)$ be a function going to zero sufficiently eagerly that $\delta<\frac{1}{K}$ and $\gamma(\pi, K)$ holds, where -here and henceforth- we abbreviate $\delta=\delta(K)$.

Centered at $G_{\sigma_{K}}$, we would like to have a ball $\Gamma_{K}$ of transformations so that for each $\lambda$ :

$$
G_{\lambda} \in \Gamma_{K} \Longrightarrow D_{\sigma_{K}}(\lambda)<\delta .
$$

So, courtesy the Frequency Lemma (14), letting

$$
\Gamma_{K}:=\mathrm{d}_{K}-\operatorname{Ball}_{\delta(K)}\left(G_{\sigma_{K}}\right)
$$

does the trick.

Handed an arbitrary $\mathcal{C}$-open set $\Upsilon \ni G_{\sigma}$, Lemma 26 assures us that the $\Gamma_{K}$ ball lies within $\Upsilon$, once $K$ is Brobdingnagian. (This uses that the $\mathrm{d}_{n}$-topologies tend to $\mathcal{C}$.) So establishing that $\wp(U)$ misses $\Gamma_{K}$ is enough to confirm $\left(25^{\prime}\right)$. In consequence, this statement:

The intersection $U^{\prime}:=\wp^{-1}\left(\Gamma_{K}\right) \cap U$ is empty,

is what we wish to substantiate.

Restating $(* *)$, if $G_{\rho} \in \wp^{-1}\left(\Gamma_{K}\right)$ then $\boldsymbol{D}_{\sigma_{K}}(\lambda)<\delta$. So property $\gamma(\pi, K)$ delivers that $G_{\rho} \notin U$. Thus the $\mathcal{C}$-dense set

$$
\left\{G_{\rho} \mid \rho \text { is an } L \text {-cycle, for some } L \triangleright K\right\}
$$

is disjoint from $U^{\prime}$. In light of the fact that $U^{\prime}$ is $\mathcal{C}$-open, it must of needs be empty.

Demonstrating $\gamma(\pi, K)$ for large $K$. Choose an $L \ngtr K$ and an $L$-cycle $\rho$. Similar to the paragraph of $(17)$, cut $\rho$ to produce a $Q_{J}$-name

$$
\mathbf{c}=c_{0} c_{1} c_{2} c_{3} \cdots c_{L-1},
$$

and let $\oplus$ mean addition modulo $L$. Recall that $1-\boldsymbol{D}_{\pi}(\rho)$ is the probability that an index $\ell \in[0 . . L)$ satisfies

$$
\pi\left(c_{\ell}\right)=c_{\ell \oplus 1},
$$

that is, is a position of agreement of the $Q_{J}$-structures of $\rho$ and $\pi$. 
Here now is the idea behind the proof of $\gamma(\pi, K)$.

(28) Lemma. For each $\varepsilon$ positive, for all large $K$, for each cycle $\rho$ with $\boldsymbol{D}_{\sigma_{K}}(\rho)<\delta$, the following holds:

$\left(28^{\prime}\right) \quad$ With probability exceeding $1-\varepsilon$, if an index $\ell$ is a position of agreement of $\rho$ with $\pi$, then $\ell \oplus K$ is a position of disagreement.

In particular, property $\gamma(\pi, K)$ holds once $\varepsilon$ is small enough.

Proof. Call a word w an "AB-block" if, using the words from $\left(26^{\prime}\right)$, it is a concatenation

$$
\mathbf{w}=\mathbf{A B A B A B} \cdots \mathbf{A B}
$$

of consecutive copies of $\mathbf{A B}$, possibly starting or ending with a partial copy.

Recall that $\mathbf{A}$ is a concatenation of copies of word $\mathrm{a}_{1} \cdots \mathrm{a}_{H}$, as $\mathbf{B}$ is of $\mathrm{b}_{1} \cdots \mathrm{b}_{H}$. Let a prime, ', flip a letter to the opposite letter having the same index; so $a_{5}^{\prime}$ means $b_{5}$, and $b_{5}^{\prime}$ means $a_{5}$.

Since words $\mathbf{A}$ and $\mathbf{B}$ each have length $K / 2$, every $\mathbf{A B}$-block $\mathbf{w}$ is antiperiodic with period $K / 2$, in this sense:

$$
w_{i+\frac{K}{2}}=w_{i}^{\prime},
$$

whenever both indices $i$ and $i+\frac{K}{2}$ are in w. where

$\sigma_{K}$-blocking the cycle $\lambda$. The $Q_{J}$-structure of $\lambda=\rho^{2}$ is $\left(\mathbf{c}^{\text {Even }}\right)\left(\mathbf{c}^{\text {Odd }}\right)$,

$$
\mathbf{c}^{\text {Even }}:=c_{0} c_{2} c_{4} c_{6} \cdots c_{L-2}, \quad \mathbf{c}^{\text {Odd }}:=c_{1} c_{3} c_{5} c_{7} \cdots c_{L-1} .
$$

Let the symbol $\mathbf{c}_{[7 . .13]}^{\text {Even }}$ mean the subsequence of $\mathbf{c}^{\text {Even }}$ with indices in the interval [7..13]. In this example,

$$
\mathbf{c}_{[7 . .15]}^{\text {Even }}=c_{8} c_{10} c_{12} c_{14} \quad \text { and } \quad \mathbf{c}_{[7 . .15]}^{\mathrm{Odd}}=c_{7} c_{9} c_{11} c_{13} c_{15},
$$

where, for $\mathbf{c}_{[7 . .15]}^{\text {Odd }}$, we have made the analogous definition.

Now suppose $K=6$. Then walking $\frac{K}{2}=3$ steps along $\mathbf{c}^{\text {Odd }}$ brings us from $c_{7}$ to $c_{13}$, i.e., to $c_{7+K}$. So the above antiperiodicity gives this generalization, for every $\ell \in[0 . . L)$ :

$$
\text { If } \mathbf{c}_{[\ell . . \ell+K]}^{\text {Even }} \text { and } \mathbf{c}_{[\ell . . \ell+K]}^{\mathrm{Odd}} \text { are each } \mathbf{A B} \text {-blocks, then } c_{\ell+K}=c_{\ell}^{\prime} .
$$

Lower-bounding disagreement. Since $\varepsilon$ and $K$ are known in advance, we can take $\delta$ small enough $\left({ }^{6}\right)$ that inequality $\boldsymbol{D}_{\sigma_{K}}(\lambda)<\delta$ implies the following: With probability exceeding $1-\varepsilon$, an index $\ell$ in $[0 . . L)$ satisfies (*) Sequences $\mathbf{c}_{[\ell . . \ell+1+K]}^{\text {Even }}$ and $\mathbf{c}_{[\ell . . \ell+1+K]}^{\text {Odd }}$ are each $\mathbf{A B}$-blocks.

$\left({ }^{6}\right)$ Letting $\delta$ be $[\varepsilon /[20 K]]^{4}$ works. This uses the argument of the Blocking Lemma, $(18 \mathrm{~b})$. 
Finally, suppose that such an $\ell$ is a position of agreement of $\rho$ with $\pi$. If $c_{\ell}$ is in $\mathbf{A}$, say $c_{\ell}=\mathrm{a}_{5}$, then $c_{\ell+1}=\pi\left(\mathrm{a}_{5}\right)=\mathrm{b}_{5}$. Consequently, by $(*)$,

$$
c_{\ell+K}=\mathrm{a}_{5}^{\prime}=\mathrm{b}_{5} \text { and } c_{\ell+1+K}=\mathrm{b}_{5}^{\prime}=\mathrm{a}_{5} .
$$

But $\pi\left(c_{\ell+K}\right)=\pi\left(\mathrm{b}_{5}\right)=\mathrm{a}_{6}$ (well. . the 6 is actually to be taken modulo $H$ ). Consequently,

$$
\pi\left(c_{\ell+K}\right) \neq \mathrm{a}_{5}=c_{\ell+1+K} .
$$

Thus position $\ell+K$ is a position of disagreement between $\rho$ and $\pi$.

A similar argument goes through if $c_{\ell}$ is in $\mathbf{B}$. Thus we have established $\left(28^{\prime}\right)$. This wraps up the proof of $(23 \mathrm{a})$ of the Complexity Theorem.

\section{$\S$ E. EGRESS}

Questions in Newtonian mechanics lead to dynamical systems in which "time is real"; the systems are $\mathbb{R}$-actions (flows $\phi: \mathbb{I} \times \mathbb{R} \rightarrow \mathbb{I}$ ) rather than the $\mathbb{Z}$-actions studied in the current article.

(Q2) Question. Does the generic transformation embed in a flow? Is the set

$$
\{T \mid T(\cdot)=\phi(\cdot, 1) \text { for some flow } \phi \text { on } \mathbb{I}\}
$$

of transformations a residual subset of $\boldsymbol{\Omega}$ ?

For such a $T$, the set of those transformations $S$ which commute with $T$, the commutant of $T$, includes a copy of $\mathbb{R}$. So an inexpensive "no" to (Q2) would follow from showing that only a meager set of $T$ have an uncountable commutant.

Alas, the generic $T$ is rank- 1 and rigid, thus necessarily has commutant which is uncountable $\left({ }^{7}\right)$.

(Q3) Question. Does the generic T embed in a (measure-preserving) $\mathbb{Q}$ action?

For a $T \in$ RAO, how close does the RAO Theorem come to answering (Q3)? Certainly, for each $n$, we can pick an $n$-th root $R_{n}$ of $T$, then fix a set $\mathcal{S} \subset[2 . . \infty)$ and look at the group $G_{\mathcal{S}} \subset \boldsymbol{\Omega}$ which is generated by $\left\{R_{n}\right\}_{n \in \mathcal{S}}$. However, the RAO theorem gives no guarantee that $R_{n}$ goes to the identity, as $n \rightarrow \infty$. So RAO does not give us control, in terms of $\mathcal{S}$ solely, on the topology of $G_{\mathcal{S}}$. This is the reason that the groups below are equipped with the "no restriction" (i.e., discrete) topology.

$\left({ }^{7}\right)$ Katok and Stepin showed, in [9], that "rank-1 and rigid" is generic, although using a different language. Definitions of rank-1 and of rigid appear in [11]. That rigidity implies uncountable commutant appears in [8] and [10]. 
Fixing $K$, let $\mathbb{Q}_{K}$ be the additive subgroup of the rationals generated by $1 / p^{K}$ as $p$ ranges over all the primes; equip $\mathbb{Q}_{K}$ with the discrete topology. Call each $p^{K}$ a "K-prime".

Evidently $\mathbb{Q}_{K}$ comprises all ratios

$$
n /\left[p_{1}^{k_{1}} p_{2}^{k_{2}} \cdots p_{J}^{k_{J}}\right]
$$

where $n$ is an arbitrary integer and each $k_{j} \in[0 . . K]$.

(29) Theorem. The generic $T$ extends to a $\mathbb{Q}_{K}$-action.

P r o of. It suffices to fix a $T$ in

$$
\text { WEAKMIXING } \cap \text { RANK- } 1 \cap \text { RAO }
$$

and extend it to a $\mathbb{Q}_{K}$-action.

For each $K$-prime $\gamma$, let $R_{\gamma}$ be a $\gamma$-th root of $T$. Let $G_{K}$ be the subgroup of $(\Omega, \circ)$ generated by the $R_{\gamma}$ transformations. Since $T$ is rank-1, its commutant is abelian $\left({ }^{8}\right)$ and thus $G_{K}$ is abelian.

To show that $G_{K}$ is isomorphic to $\mathbb{Q}_{K}$, define a map $\psi$ from $\left(G_{K}, \circ\right)$ to $\left(\mathbb{Q}_{K},+\right)$ as follows. For each finite set of $K$-primes $\{\alpha, \beta, \ldots, \gamma\}$, and of integers $a, b, \ldots, c$, let

$$
\psi\left(R_{\alpha}^{a} \circ R_{\beta}^{b} \circ \cdots \circ R_{\gamma}^{c}\right):=\frac{a}{\alpha}+\frac{b}{\beta}+\cdots+\frac{c}{\gamma} .
$$

That this $\psi$ is a group isomorphism will follow immediately once $\psi$ is shown to be well defined.

To address this latter aim, suppose that exponents $a, \ldots, c$ cause $S:=R_{\alpha}{ }^{a} \circ \cdots \circ R_{\gamma}{ }^{c}$ to be the identity transformation. Let $L$ be the product $\alpha \cdots \gamma$; then $S^{L}=T^{k}$, where

$$
k:=a \frac{L}{\alpha}+\cdots+c \frac{L}{\gamma} \stackrel{\text { note }}{=} L \cdot\left[\frac{a}{\alpha}+\cdots+\frac{c}{\gamma}\right] .
$$

Since $T$ is weak-mixing it cannot be periodic, so $k$ must be zero. Thus $\frac{a}{\alpha}+\cdots+\frac{c}{\gamma}$ is zero.

Root chains. Related to (Q3) is this: Does the generic $T$ extend to a $\mathbb{Q}_{\infty}$-action? Here, $\mathbb{Q}_{\infty}$ is the group of rationals $(\mathbb{Q},+)$, but equipped with the discrete topology.

In light of the foregoing theorem, what is the obstruction to fabricating a $\mathbb{Q}_{\infty}$-action? If, for each prime $p$, we can produce an infinite length " $p$-chain"

$$
T=: S_{0} \underset{p}{\leftarrow} S_{1} \underset{p}{\leftarrow} S_{2} \underset{p}{\leftarrow} \ldots
$$

$\left({ }^{8}\right)$ Although this follows from the Weak-Closure theorem, [10], there is a generic subset of RANK-1 -the maps with "flat stacks" - where abelianness of the commutant follows by an elementary argument. 
where each $S_{k+1}$ is a $p$-th root of $S_{k}$, then a $\mathbb{Q}_{\infty}$-action can be built as above. Thus one is led to ask:

(Q4) Question. Does there exist a weak-mixing $T$ which has square-root chains of each finite length, but no infinite square-root chain?

I raised this question at the Ergodic Theory seminar while on sabbatical at U. of Toronto, in 1996/7. The menagerie of examples and techniques from the 1960/70's suggested a "yes" to (Q4). One natural approach, harking back to Ornstein's construction, is the "counterexample machine" built by Dan Rudolph, which uses the rank-1 mixing map of Ornstein.

Vaguely, Rudolph's machine takes a permutation of $\mathbb{Z}$, and produces a weak-mixing transformation with analogous properties. A standard approach to (Q4), then, would be to search the group of $\mathbb{Z}$-permutations for:

A permutation $\pi$ of $\mathbb{Z}$ which has arbitrarily long square-root chains, but no infinite chains.

Alas, the reader can verify that no such $\pi$ exists.

Techniques of del Junco and others. Nonetheless, ideas of the counterexample machine can be used. Andrés del Junco developed machinery, for certain abelian groups $G$, which produces a $G$-action $\phi: \mathbb{I} \times G \rightarrow \mathbb{I}$ for which the commutant of certain transformations in the action is limited to the $G$-action itself.

This suggested first constructing a denumerable abelian group $(M, \boxplus, e)$ and an element $\eta \in M$ such that $\eta$ has square-root chains of each finite length, but has no infinite chain. Here is one such group, the Madore group:

Let $M$ be the free abelian group on symbols (generators) $\eta, \gamma_{1}, \gamma_{2}, \ldots$, where the generating relations are

$$
\underbrace{\gamma_{j} \boxplus \gamma_{j} \boxplus \ldots \boxplus \gamma_{j}}_{2^{j} \text { copies }}=\eta, \quad \text { for } j=1,2, \ldots
$$

We now describe $M$ in an alternative way.

Let $G_{j}$ be the additive cyclic group $\left[0 . .2^{j}\right)$; that is, $\mathbb{Z} / 2^{j} \mathbb{Z}$. As a set, define $M$ to be the direct sum

$$
M:=\mathbb{Z} \oplus G_{1} \oplus G_{2} \oplus \ldots
$$

So $M$ comprises all tuples $\left\langle a \mid g_{1}, g_{2}, \ldots\right\rangle$ where $a \in \mathbb{Z}$ and $g_{j} \in G_{j}$, and only finitely many of the $g_{j}$ are non-zero. Given $\alpha:=\left\langle a \mid g_{1}, g_{2}, \ldots\right\rangle$ and $\beta:=\left\langle b \mid h_{1}, h_{2}, \ldots\right\rangle$, let $N$ be smallest natural number with $g_{n}=h_{n}=0$, 
for all $n>N$. Define addition in $M$ by

$$
\alpha \boxplus \beta:=\left\langle a+b+\sum_{j=1}^{N} c_{j} \mid r_{1}, r_{2}, \ldots\right\rangle,
$$

where $c_{j}$ is the $j$-th "carry" in group $\left[0 . .2^{j}\right)$, and $r_{j}$ is the remainder. That is,

$$
g_{j}+h_{j}=r_{j}+2^{j} c_{j}, \quad \text { where } r_{j} \in\left[0 . .2^{j}\right) \text { and } c_{j} \text { is either } 0 \text { or } 1 .
$$

Thus $\boxplus$ is component-wise addition, with a carry from the $j$-th component into the zero-th component. Evidently $M$ is abelian with $e:=\langle 0 \mid 0, \ldots\rangle$ its neutral element.

To write down an additive inverse, $\boxminus \alpha$, let $J$ be the number of indices $j$ with $g_{j} \neq 0$. Then

$$
\boxminus \alpha=\left\langle-[a+J] \mid f_{1}, f_{2}, f_{3}, \ldots\right\rangle,
$$

where $f_{j}$ is the $G_{j}$-inverse of $g_{j}$. So $f_{j}$ is $2^{j}-g_{j}$ if $g_{j} \neq 0$, and is zero otherwise.

Identify $\langle k \mid 0, \ldots\rangle$ with the integer $k$, thus exhibiting a copy of $(\mathbb{Z},+)$ inside of $(M, \boxplus)$. Our $M$ is generated by the collection $\left\{\eta, \gamma_{1}, \gamma_{2}, \ldots\right\}$, where

$$
\begin{aligned}
\eta & :=\langle 1 \mid 0,0,0,0,0,0, \ldots\rangle \quad \text { The } 1 \text { is in the zero-th position. } \\
\gamma_{j} & :=\langle 0 \mid 0, \ldots, 0,1,0, \ldots\rangle \quad \text { This } 1 \text { is in the } j \text {-th position. }
\end{aligned}
$$

Since $\gamma_{j}$ is a $2^{j}$-th root of $\eta$, one sees that (33) with operation $\boxplus$ is the same group as defined by (32).

Fixing $j$, there is a square-root chain

$$
\eta<2^{j-1} \gamma_{j}<2^{j-2} \gamma_{j}<\ldots<4 \gamma_{j}<2 \gamma_{j}<\gamma_{j}
$$

of length $j$. Yet one can check that no element in $M \backslash\{e\}$ has an infinite chain.

Answering "yes" to (Q4). Blair Madore, a student of del Junco, proved the following theorem as part of his doctoral travails.

(34) MAdore's Theorem (Theorem 1.1 in [13]). Let $G$ be a countable abelian group with subgroup $\mathbb{Z}^{d}$, for some $d \geq 1$, where each element of the quotient group $G / \mathbb{Z}^{d}$ has finite order. Then there exists a rank-one action of $G$ so that the transformation $T$ corresponding to $(1,0,0, \ldots, 0)$ in $\mathbb{Z}^{d}$ is mixing, simple, and only commutes with the other transformations in $G$.

In particular, there is such an action $\phi$ of the Madore group. With $\eta$ meaning $\langle 1 \mid 0,0 \ldots\rangle$, then, $T:=\phi^{\eta}$ is a mixing transformation having no infinite square-root chain. 
Conditions implying a $\mathbb{Q}_{\infty}$ extension. Various general results would imply that the generic $\mathbb{Z}$-action extends to an action of the discrete rationals.

(Q5) Question. Generically, does T have at most one square-root?

If a map $T$ in (30) had, for each $j$, at most one $j$-th root, then the proof of (29) would extend $T$ to a unique $\mathbb{Q}_{\infty}$-action.

Note that (Q5) is equivalent to this query: For the generic rank-1 map T, is the identity map the only involution commuting with $T$ ?

Closing thoughts. The RAO theorem was proved under the aegis of the coarse topology on $\boldsymbol{\Omega}$. Because the set of maps with RAO is an analytic set, the Equivalence Theorem of [4, Thm. 7] applies to say that RAO is also residual in another standard setting, the Polish space of shift-invariant Borel probability measures on the Hilbert cube $[0,1]^{\mathbb{Z}}$.

It may well turn out that there are more general equivalence theorems, and that settings whose topology is more natural for the transformations arising in physics nonetheless have the same abstract genericity properties.

\section{REFERENCES}

[1] F. Beleznay, The complexity of the collection of countable linear orders of the form $I+I$, preprint, 1997.

[2] K. R. Berg, Mixing, cyclic approximation, and homomorphisms, Maryland Technical Report, 1975.

[3] N. Friedman, P. Gabriel, and J. L. King, An invariant for rigid rank-1 transformations, Ergodic Theory Dynam. Systems 8 (1988), 53-72.

[4] E. Glasner and J. L. King, A zero-one law for dynamical properties, in: Topological Dynamics and Applications (Minneapolis, MN, 1995), Amer. Math. Soc., Providence, RI, 1998, 231-242.

[5] P. R. Halmos, Lectures on Ergodic Theory, Publ. Math. Soc. Japan 3, Math. Soc. Japan, 1956.

[6] P. D. Humke and M. Laczkovich, The Borel structure of iterates of continuous functions, Proc. Edinburgh Math. Soc. (2) 32 (1989), 483-494.

[7] A. del Junco and D. Rudolph, On ergodic actions whose self-joinings are graphs, Ergodic Theory Dynam. Systems 7 (1987), 531-557.

[8] A. B. Katok, Ya. G. Sina ̌, and A. M. Stepin, The theory of dynamical systems and general transformation groups with invariant measure, (errata insert), Vsesoyuz. Inst. Nauchn. i Tekhn. Informatsii, Akad. Nauk SSSR, Moscow, 1975, 129-262 (in Russian).

[9] A. B. Katok and A. M. Stepin, Approximations in ergodic theory, Uspekhi Mat. Nauk 22 (1967), no. 5, 81-106.

[10] J. L. King, The commutant is the weak closure of the powers, for rank-1 transformations, Ergodic Theory Dynam. Systems 6 (1986), 363-384.

[11] - Joining-rank and the structure of finite rank mixing transformations, J. Anal. Math. 51 (1988), 182-227.

[12] K. Kuratowski, Topology, Vol. I, Academic Press, New York, 1966. 
[13] B. F. Madore, Rank-one mixing actions with simple $\mathbb{Z}$ subactions, Doctoral dissertation, 2000.

[14] D. S. Ornstein, On the root problem in ergodic theory, in: Proc. Sixth Berkeley Sympos. Math. Statist. Probab. (Berkeley, CA, 1970/1971), Vol. II: Probability Theory, Univ. California Press, Berkeley, CA, 1972, 347-356.

[15] D. J. Rudolph, An example of a measure preserving map with minimal selfjoinings, and applications, J. Anal. Math. 35 (1979), 97-122.

[16] W. A. Veech, A criterion for a process to be prime, Monatsh. Math. 94 (1982), $335-341$.

University of Florida

PO Box 118105

Gainesville, FL 32611-2082, U.S.A.

E-mail: squash@math.ufl.edu

Web: http:/www.math.ufl.edu/ $\sim$ squash/ 